\title{
Preface
}

\section{Thoracic Transplantation}

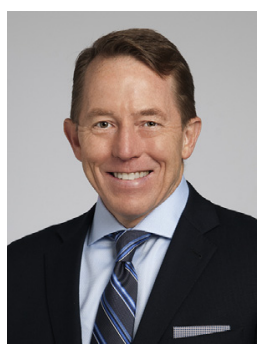

Kenneth R. McCurry, MD

Editor

Millions of people around the world suffer from chronic respiratory or chronic heart failure that could potentially benefit from lung or heart transplantation, respectively. While epidemiologically still small, the number of patients receiving thoracic transplants has grown immensely over the last 50 years. Through June 2016, more than 60,000 adult lung, 3900 adult heart-lung, and 135,000 adult heart transplants have been reported to the International Society for Heart and Lung Transplantation worldwide. In the United States alone, in 2016, more than 2300 adult lung transplants and more than 2700 adult heart transplants were performed. As a result, in this issue of Critical Care Clinics, we have focused on the pretransplant and posttransplant critical care of patients undergoing thoracic transplantation. While ventricular assist devices are commonly utilized to bridge patients to heart transplantation, as well as for destination therapy, we have not discussed this therapy in this issue as this was the focus of a recent issue of Critical Care Clinics.

In one of the articles, I provide some historical context to how we arrived at our current day approach to thoracic transplantation and outline some of the problems that are the focus of subsequent articles. As in many other areas of medicine, and surgery in particular, the pioneering physicians recognized need and demonstrated vision and (perhaps most importantly) extreme persistence in the face of failure to make these therapies the mainstream treatment options they are today. Of course, none of these therapies would be possible without the courage of those initial patients who put their lives in the hands of others for the hope of a longer and better life.

Dr Mattar, Dr Chatterjee, and Dr Loor (Texas Heart Institute at Baylor St. Luke's) highlight the rapidly evolving field of bridging to lung transplantation. While historically thought to be a poor option with poor outcomes, bridging to lung transplantation with an extracorporeal circuit has recently been increasingly utilized to support patients to lung transplantation. They provide an overview of published outcomes and very 
specific recommendations on selection of patients, type of extracorporeal circuit and management, and decision-making guidelines.

Dr Geube, Dr Anandamurthy, and Dr Yared (Cleveland Clinic) discuss the complex challenge of managing the lung transplant recipient in the perioperative period. They discuss the new grading system for perioperative allograft dysfunction (primary graft dysfunction), define modifiable and unmodifiable risk factors, and provide a pragmatic and comprehensive management strategy that has applicability to other forms of acute lung injury.

Dr Kaczorowski and Dr Rabin (University of Maryland) provide an up-to-date review of the issues involved and management strategies utilized in the perioperative phase following heart transplantation. They also describe the evolving field of mechanical circulatory support and options for medically refractory cardiac graft failure.

Dr Puttarajappa, Dr Bernado, and Dr Kellum (University of Pittsburgh) reflect on the common complication of renal insufficiency following thoracic transplantation. While outlining organ-specific differences, they describe the epidemiology, risk factors, pathogenesis, implications, and management and make very specific recommendations on ways to avoid further renal injury.

Dr Husain and Dr Alsaeed (University of Toronto) provide a comprehensive overview of infections seen in thoracic transplant recipients. Importantly, they provide information on the changing epidemiology of infections in this population and describe the sometimes atypical presentation that clinicians should be aware of.

I am extremely grateful to the authors for their contributions. Collectively, we hope that this issue is helpful and provides useful information to assist with patient care.

Kenneth R. McCurry, MD Department of Thoracic and Cardiovascular Surgery Heart and Vascular Institute and

Lerner Research Institute

Cleveland Clinic

9500 Euclid Avenue, J4-1 Cleveland, $\mathrm{OH}$ 44195, USA 